\title{
Belphégor
}

Littérature populaire et culture médiatique

16-1 | 2018

Hommage à Charles Grivel

\section{Nyela, Désiré. La Filière noire (Dynamiques du polar " made in Africa »).}

Sándor Kálai

\section{(2) OpenEdition}

1 Journals

\section{Electronic version}

URL: https://journals.openedition.org/belphegor/1171

DOI: 10.4000/belphegor.1171

ISSN: 1499-7185

Publisher

LPCM

\section{Electronic reference}

Sándor Kálai, “Nyela, Désiré. La Filière noire (Dynamiques du polar « made in Africa »).", Belphégor [Online], 16-1 | 2018, Online since 16 July 2018, connection on 19 October 2021. URL: http:// journals.openedition.org/belphegor/1171 ; DOI: https://doi.org/10.4000/belphegor.1171

This text was automatically generated on 19 October 2021

\section{(c) (i) (9)}

Belphégor est mis à disposition selon les termes de la Licence Creative Commons Attribution - Pas d'Utilisation Commerciale - Pas de Modification 4.0 International. 
Nyela, Désiré. La Filière noire (Dynamiques du polar « made in Africa»).

Sándor Kálai

\section{REFERENCES}

Nyela, Désiré. La Filière noire (Dynamiques du polar « made in Africa »). Paris: Honoré Champion, 2015. 280 p. ISBN 9782745329523. 


\section{La Filière noire}

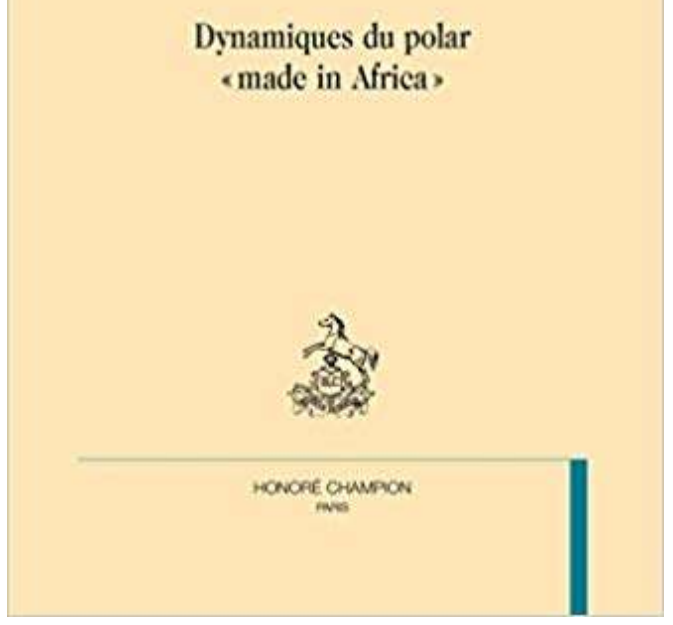

1 Depuis plus d'un siècle le récit policier, dans ses différents avatars génériques et médiatiques, se caractérise par un foisonnement extraordinaire. Ces dernières décennies on peut constater dans l'histoire du genre une sorte de tournant 'spatial': ce n'est pas tant la représentation du crime et de ses conséquences qui importe, mais plutôt le lieu où un crime se produit. C'est ce qui peut expliquer à la fois des tendances comme la popularité du polar scandinave ou méditerranéen ou l'augmentation continue du nombre des traductions de polars sur les différents marchés du livre nationaux. Le polar n'apparaît donc pas seulement comme une importante activité commerciale, mais aussi comme un puissant moyen de saisir la réalité sociale.

C'est dans ce contexte que l'important livre de Désiré Nyela s'inscrit. L'auteur, qui enseigne à l'Université de Sainte-Anne, en Nouvelle-Écosse, au Canada, a fait ses études universitaires à Yaoundé, au Cameroun, et a soutenu sa thèse à l'Université de ParisSorbonne (Paris IV). Il est spécialiste de la sémiostylistique, des littératures francophones (Afrique, Maghreb, Caraïbes, Europe) et des littératures populaires (roman policier, roman d'espionnage, roman sentimental). Son livre intitulé La Filière noire se consacre à l'étude de l'histoire du genre policier sur le continent africain. Désiré Nyela s'intéresse donc à une forme littéraire doublement minoritaire, le genre policier africain, minoritaire à l'intérieur de la littérature africaine elle-même, encore souvent considérée, de nos jours, comme littérature minoritaire.

Les lecteurs assidus de polars, aussi bien 'amateurs' que 'professionnels', peuvent avoir des connaissances sur le roman policier africain avant même d'ouvrir le livre - mais ces connaissances ne se limitent, sans doute, qu'à quelques auteurs, comme Deon Meyer, ou à quelques pays (Afrique de Sud). On peut parler d'une absence relative du polar en Afrique et le livre de Désiré Nyela veut nuancer cette constatation: il propose de délimiter le corpus à l'Afrique sub-saharienne en choisissant des auteurs tant 
anglophones que francophones (ainsi le polar maghrébin, avec Yasmina Khadra, est exclu de ce parcours).

4 L'analyse, qui se veut à la fois descriptive (donner « l'inventaire le plus représentatif possible du corpus»(31)), historique (esquisser "l'histoire d'un genre dans un continent jadis dépourvu de tradition » (31)) et esthétique (examiner "les nuances et teintes particulières qui ressortent et singularisent ces polaristes » (31)) est encadrée par deux chapitres théoriques. L'introduction esquisse les problèmes fondamentaux d'une littérature africaine qui se caractérise par une double filiation: filiation culturelle, parce que cette littérature est issue de la culture traditionnelle, et filiation générique, parce qu'elle est déterminée par l'héritage colonial. D'où son caractère second, qui manifeste la dépendance de l'Afrique vis-à-vis des institutions culturelles des anciennes puissances colonisatrices. Ainsi, le choix du polar, un genre populaire issu de l'Occident, ne semble pas de prime abord un bon choix pour les auteurs africains. Ces derniers se trouvent aussi dans une situation paradoxale, étant donné le fait que la place éditoriale privilégiée du polar en français est Paris: les auteurs sont doublement étrangers, à la fois par rapport à leur culture et par rapport aux institutions occidentales.

Les différents chapitres du livre retracent la dynamique du polar africain du moment des premières hésitations jusqu'à l'affirmation du genre - même si le parcours ne respecte pas entièrement la chronologie. Le premier chapitre s'intéresse aux premières manifestations du genre au milieu des années 1980. Il évoque notamment les romans de trois auteurs différents, tous parus dans la collection (malheureusement éphémère) de L'Harmattan, « Polar Noir »: Arturo Junior Nzau: Traite au Zaïre (1984), Evina Abossolo: Cameroun/Gabon. Le D.A.S.S. monte à l'attaque (1985) et Asse Gueye: No woman no cry (1986). Les noms d'auteur et les titres annoncent d'une part une variété géographique, et d'autre part un mélange de deux genres très proches, le récit policier et le récit d'espionnage. Désiré Nyela parle de l'échec de la collection, dû à des instabilités: la police ne joue qu'un rôle mineur. Il faut également relever l'absence de la sérialité, un vecteur important dans l'institutionnalisation du genre.

6 Après cette première phase d'hésitation, vient le moment de l'appropriation des codes génériques. Cela ne peut se passer que par le jeu intertextuel, lequel constitue un des aspects textuels de la conscience du genre, mais cette seconde époque se caractérise aussi par une institutionnalisation plus aboutie (l'institutionnalisation incarnant l'aspect social de la conscience du genre). Le roman de Simon Njami (Cercueil et Cie.) reprend les personnages de Chester Himes, un modèle du genre; Boya Baenga et Achille Ngoye poursuivent une veine réaliste et sociologique. L'archer bassari de Modibo Sounkalo Keita est le premier polar entièrement ancré dans la réalité du continent. Dans ce chapitre une place à part revient à Moussa Konaté (un des dédicataires du livre): le commissaire Habib est un des rares héros sériel du polar africain, un flic philosophe qui travaille avec son fidèle adjoint, Sosso. Dans L'Assassin de Banconi, le commissaire a affaire à une population façonnée par l'Islam; dans L'honneur des Keita il s'agit essentiellement de la lutte entre deux forces, la modernité, incarnée par le commissaire, et le pouvoir ancestral, représenté par le chef des Keita. Certains récits de Konaté peuvent être considérés comme des polars ethnologiques qui supposent aussi une sensibilité interculturelle non seulement dans le processus de l'enquête, mais aussi dans l'acte de lecture. Ces enquêtes permettent à Habib (mais aussi au lecteur, qui est sans doute un lecteur sériel) d'en savoir toujours un peu plus sur les Maliens, mais aussi 
sur lui-même. On peut aussi constater, au terme des analyses de ce chapitre, que l'appropriation des codes du genre est parallèle à l'entrée de certains auteurs dans des collections prestigieuses: « Série Noire " pour Konaté, Abasse Ndione, Achille Ngoye et Aïda Mady Diallo ou « Fayard noir » pour Konaté.

7 Le chapitre trois - qui porte comme titre Affirmation - rompt avec la logique chronologique. Ce titre est à entendre dans deux sens. Il s'agit d'une part d'évoquer la dynamique d'affirmation du genre, mais aussi celle d'œuvres plus féminines: les analyses de ce chapitre s'intéressent soit aux personnages, soit aux écrivaines de polar africaines. L'auteur consacre un sous-chapitre au premier roman de Kwei Quartey, Wife of the Gods. Le cantique des cannibales de Florent Couao-Zotti met en scène une figure féminine atypique, Gloh, reine des bandits. Mais il s'agit surtout, dans cet ensemble, de deux séries. Il se penche d'une part sur le personnage d'Alexander McCall Smith, Mma Ramotswe, détective: cette propriétaire de la seule agence de détectives au féminin du Botswana est l'incarnation de la tradition (par son aspect physique) et elle s'inscrit en même temps dans la modernité par son féminisme ancré dans l'action. Ensuite Désiré Nyela consacre un sous-chapitre à la série des aventures de Claire Hart, profileuse créée par Margie Orford, romancière sud-africaine d'origine anglaise dont les romans en traduction française sont publiés par Payot et Rivages.

8 Le dernier chapitre, intitulé Confirmation, est consacré au polar du Sud, essentiellement sud-africain. Les deux derniers romans de Mongo Beti, auteur camerounais, (deux romans d'une trilogie restée inachevée) offrent l'exemple d'une trajectoire d'écrivain qui n'est plus surprenante (du moins dans la littérature occidentale), où les frontières entre littérature populaire et littérature majusculée se trouvent brouillées. LouisFerdinand Despreez, descendant de huguenots français, qui confronte dans ses romans policiers ses personnages à l'Histoire et à ses refoulés, Roger Smith, qui s'intéresse, entre autres, aux personnages de métis, Wessel Ebersohn, avec son psychologue d'origine juive, Yudel Gordon, ou les romans de Deon Meyer avec un héros collectif, la police du Cap - proposent tous la représentation d'un pays en profonde mutation, l'Afrique du Sud. Ces romans témoignent du fait que les victimes sont souvent des enfants et des femmes et dans leur traitement de la violence, le supplice infligé au corps renvoie aux turpitudes de la société.

L'introduction de l'ouvrage a évoqué le cas de Yambo Ouologuem et de son roman, Le Devoir de violence, comme exemple d'une littérature africaine très différente par rapport à celle des représentants de la négritude. Ce roman invite à exercer un regard différent, qui ne vient pas d'en haut, mais s'effectue à partir des marges et aboutit à «la phénoménologie d'un regard sur soi » (25). La conclusion semble reprendre, en quelque sorte, cette réflexion, parce que le polar africain s'inscrit dans la dynamique d'un regard critique sur soi: il s'agit de la perception d'un continent vu de l'intérieur et à travers le prisme de la marginalité criminelle. En s'intéressant au fonctionnement du discours du polar, l'auteur propose un éclairage sur le genre. Le roman policier pour l'auteur africain devient ainsi un "genre approprié pour [...] dire la tragique condition » (263) de l'homme. En ce sens, Désiré Nyela nous invite à porter un regard différent sur la littérature africaine. 


\section{AUTHOR}

SÁNDOR KÁLAI

Université de Debrecen 